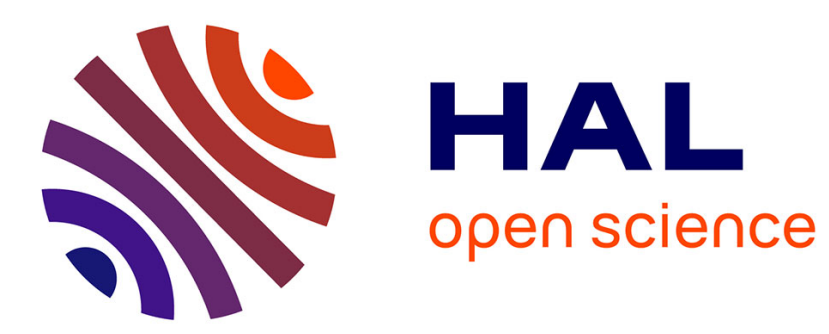

\title{
Enumerating the edge-colourings and total colourings of a regular graph
}

Stéphane Bessy, Frédéric Havet

\section{To cite this version:}

Stéphane Bessy, Frédéric Havet. Enumerating the edge-colourings and total colourings of a regular graph. Journal of Combinatorial Optimization, 2013, 25 (4), pp.523-535. 10.1007/s10878-011-9448-5 . hal-00821598

\section{HAL Id: hal-00821598 \\ https://hal.inria.fr/hal-00821598}

Submitted on 23 Oct 2016

HAL is a multi-disciplinary open access archive for the deposit and dissemination of scientific research documents, whether they are published or not. The documents may come from teaching and research institutions in France or abroad, or from public or private research centers.
L'archive ouverte pluridisciplinaire HAL, est destinée au dépôt et à la diffusion de documents scientifiques de niveau recherche, publiés ou non, émanant des établissements d'enseignement et de recherche français ou étrangers, des laboratoires publics ou privés. 


\title{
Enumerating the edge-colourings and total colourings of a regular graph* $^{*}$
}

\author{
S. Bessy ${ }^{\dagger}$ and F. Havet ${ }^{\ddagger}$
}

December 24, 2011

\begin{abstract}
In this paper, we are interested in computing the number of edge colourings and total colourings of a connected graph. We prove that the maximum number of $k$-edge-colourings of a connected $k$-regular graph on $n$ vertices is $k \cdot((k-1) !)^{n / 2}$. Our proof is constructive and leads to a branching algorithm enumerating all the $k$-edge-colourings of a connected $k$-regular graph in time $O^{*}\left(((k-1) !)^{n / 2}\right)$ and polynomial space. In particular, we obtain a algorithm to enumerate all the 3 -edge-colourings of a connected cubic graph in time $O^{*}\left(2^{n / 2}\right)=O^{*}\left(1.4143^{n}\right)$ and polynomial space. This improves the running time of $O^{*}\left(1.5423^{n}\right)$ of the algorithm due to Golovach et al. [12]. We also show that the number of 4-total-colourings of a connected cubic graph is at most $3 \cdot 2^{3 n / 2}$. Again, our proof yields a branching algorithm to enumerate all the 4-total-colourings of a connected cubic graph.
\end{abstract}

\section{Introduction}

We refer to [6] for standard notation and concepts for graphs. In this paper, all the considered graphs are loopless, but may have parallel edges. A graph with no parallel edges is said to be simple. Let $G$ be a graph. We denote by $n(G)$ the number of vertices of $G$, and for each integer $k$, we denote by $n_{k}(G)$ the number of degree $k$ vertices of $G$. Often, when the graph $G$ is clearly understood, we abbreviate $n(G)$ to $n$ and $n_{k}(G)$ to $n_{k}$. Throughout the paper, we are concerned with connected graphs.

Graph colouring is one of the classical subjects in graph theory. See for example the book of Jensen and Toft [14]. From an algorithmic point of view, for many colouring type problems, like vertex colouring, edge colouring and total colouring, the existence problem asking whether an input graph has a colouring with an input number of colours is NP-complete. Even more, these colouring problems remain NP-complete when the question is whether there is a colouring of the input graph with a fixed (and greater than 2) number of colours [11, 13, 19].

Exact algorithms to solve NP-hard problems are a challenging research subject in graph algorithms. Many papers on exact exponential time algorithms have been published in the last decade. One of the major results is the $O^{*}\left(2^{n}\right)$-time inclusion-exclusion algorithm to compute the chromatic

\footnotetext{
${ }^{*}$ This work is supported by the French Agence Nationale de la Recherche under reference AGAPE ANR-09-BLAN0159 .

${ }^{\dagger}$ Université Montpellier 2 - CNRS, LIRMM. e-mail: Stephane.Bessy@lirmm.fr.

${ }^{\ddagger}$ Projet Mascotte, I3S (CNRS, UNSA) and INRIA, Sophia Antipolis. email: Frederic.Havet@inria.fr.
} 
number of a graph found independently by Björklund, Husfeldt [3] and Koivisto [16], see [5]. This approach may also be used to establish a $O^{*}\left(2^{n}\right)$-time algorithm to count the $k$-colourings and to compute the chromatic polynomial of a graph. It also implies a $O^{*}\left(2^{m}\right)$-time algorithm to count the $k$-edge-colourings and a $O^{*}\left(2^{n+m}\right)$-time algorithm to count the $k$-total-colourings of a given graph.

Since edge colouring and total colouring are particular cases of vertex colouring, a natural question is to ask if faster algorithms than the general one may be designed in these cases. For instance, very recently Björklund et al. [4] showed how to detect whether a $k$-regular graph admits a $k$-edge-colouring in time $O^{*}\left(2^{(k-1) n / 2}\right)$.

The existence problem asking whether a graph has a colouring with a fixed and small number $k$ of colours also attracted a lot of attention. For vertex colourability the fastest algorithm for $k=3$ has running time $O^{*}\left(1.3289^{n}\right)$ and was proposed by Beigel and Eppstein [2], and the fastest algorithm for $k=4$ has running time $O^{*}\left(1.7272^{n}\right)$ and was given by Fomin et al. [10]. They also established algorithms for counting $k$-vertex-colourings for $k=3$ and 4 . The existence problem for a 3 -edge-colouring is considered in $[2,17,12]$. Kowalik [17] gave an algorithm deciding if a graph is 3 -edge-colourable in time $O^{*}\left(1.344^{n}\right)$ and polynomial space and Golovach et al. [12] presented an algorithm counting the number of 3 -edge-colourings of a graph in time $O^{*}\left(3^{n / 6}\right)=O^{*}\left(1.201^{n}\right)$ and exponential space. Golovach et al. [12] also showed a branching algorithm to enumerate all the 3edge-colourings of a connected cubic graph in time $O^{*}\left(25^{n / 8}\right)=O^{*}\left(1.5423^{n}\right)$ and polynomial space. In particular, this implies that every connected cubic graph of order $n$ has at most $O\left(1.5423^{n}\right) 3$ edge-colourings. They give an example of a connected cubic graph of order $n$ having $\Omega\left(1.2820^{n}\right)$ 3 -edge-colourings. In Section 2, we prove that a connected cubic graph of order $n$ has at most $3 \cdot 2^{n / 2}$-edge-colourings and give an example reaching this bound. Our proof can be translated into a branching algorithm to enumerate all the 3-edge-colourings of a connected cubic graph in time $O^{*}\left(2^{n / 2}\right)=O^{*}\left(1.4143^{n}\right)$ and polynomial space. Furthermore, we extend our result proving that every $k$-regular connected graph of order $n$ admits at most $k \cdot((k-1) !)^{n / 2} k$-edge-colourings. This improves the bound derived from the maximal number of perfect matchings of a graph with a given degree sequence given by Alon and Friedland [1]. Also, similarly 3-edge-colourings, we derive a branching algorithm to enumerate all the $k$-edge-colourings of a connected $k$-regular graph in time $O^{*}\left(((k-1) !)^{n / 2}\right)$ and polynomial space.

Regarding total colouring, very little has been done. Golovach et al. [12] showed a branching algorithm to enumerate the 4 -total-colourings of a connected cubic graph in time $O^{*}\left(2^{13 n / 8}\right)=$ $O^{*}\left(3.0845^{n}\right)$, implying that the maximum number of 4-total-colourings in a connected cubic graph of order $n$ is at most $O^{*}\left(2^{13 n / 8}\right)=O^{*}\left(3.0845^{n}\right)$. In Section 3 , we lower this bound to $3 \cdot 2^{3 n / 2}=$ $O\left(2.8285^{n}\right)$. Again, our proof yields a branching algorithm to enumerate all the 4 -total-colourings of a connected cubic graph in time $O^{*}\left(2.8285^{n}\right)$ and polynomial space.

\section{$2 \quad$ Edge colouring}

A (proper) edge colouring of a graph is a colouring of its edges such that two adjacent edges receive different colours. An edge colouring with $k$ colours is a $k$-edge-colouring. We denote by $c_{k}(G)$ the number of $k$-edge-colourings of a graph $G$. 


\subsection{General bounds for $k$-regular graphs}

In this section, we are interested in computing the number of $k$-edge-colourings of $k$-regular connected graphs. We start by computing exactly the number of 3-edge-colourings of the cycles.

Proposition 1. Let $C_{n}$ be the cycle of length $n$.

$$
c_{3}\left(C_{n}\right)= \begin{cases}2^{n}+2, & \text { if } n \text { is even }, \\ 2^{n}-2, & \text { if } n \text { is odd. }\end{cases}
$$

Proof. By induction on $n$. It is easy to check that $c_{3}\left(C_{2}\right)=c_{3}\left(C_{3}\right)=6$.

Let $C_{n}=\left(v_{1}, v_{2}, \ldots, v_{n}, v_{1}\right)$. Let $A$ be the set of 3-edge-colourings of $C_{n}$ such that $c\left(v_{n-1} v_{n}\right) \neq$ $c\left(v_{1} v_{2}\right)$ and $B$ the set of 3-edge-colourings of $C_{n}$ such that $c\left(v_{n-1} v_{n}\right)=c\left(v_{1} v_{2}\right)$. The 3-edgecolourings of $A$ are in one-to-one correspondence with those of $C_{n-1}$ and the pair of colourings of $B$ agreeing everywhere except on $v_{n} v_{1}$ are in one-to-one correspondence with the 3 -edge-colourings of $C_{n-2}$. Thus $c_{3}\left(C_{n}\right)=c_{3}\left(C_{n-1}\right)+2 c_{3}\left(C_{n-2}\right)$. Hence, if $n$ is even, then $c_{3}\left(C_{n}\right)=2^{n-1}-2+$ $2\left(2^{n-2}+2\right)=2^{n}+2$, and if $n$ is odd, then $c_{3}\left(C_{n}\right)=2^{n-1}+2+2\left(2^{n-2}-2\right)=2^{n}-2$.

Let us now present our method which is based on a classical tool: $(s, t)$-ordering.

Definition 2. Let $G$ be a graph and $s$ and $t$ be two distinct vertices of $G$. An $(s, t)$-ordering of $G$ is an ordering of its vertices $v_{1}, \ldots, v_{n}$ such that $s=v_{1}$ and $t=v_{n}$, and for all $1<i<n, v_{i}$ has a neighbour in $\left\{v_{1}, \ldots, v_{i-1}\right\}$ and a neighbour in $\left\{v_{i+1}, \ldots, v_{n}\right\}$.

Lemma 3 (Lempel et al. [18]). A graph $G$ is a 2-connected graph if, and only if, for every pair $(s, t)$ of vertices, it admits an $(s, t)$-ordering.

In fact, Lempel et al. established Lemma 3 only for simple graphs but it can be trivially extended to graphs since replacing all the parallel edges between two vertices by a unique edge does not change the connectivity.

Theorem 4. Let $G$ be a 2-connected subcubic graph. Then $c_{3}(G) \leq 3 \cdot 2^{n-\frac{n_{3}}{2}}$.

Proof. If $G$ is a cycle, then the result follows from Proposition 1 . Hence we may assume that $G$ is not a cycle and thus has at least two vertices of degree 3, say $s$ and $t$. By Lemma 3, there exists an $(s, t)$-ordering $v_{1}, v_{2}, \ldots, v_{n}$ of $G$. Orient each edge of $G$ according to this order, that is from the lower-indexed end-vertex towards its higher-indexed one. Let us denote by $D$ the obtained digraph. Observe that $d^{+}\left(v_{1}\right)=3=d^{-}\left(v_{n}\right)$ and $d^{-}\left(v_{1}\right)=0=d^{+}\left(v_{n}\right)$. Let $A^{+}$(resp. $A^{-}$) be the set of vertices with outdegree 2 (resp. indegree 2) in $D$ and $A_{2}$ be the set of vertices with degree 2 in $G$ (and thus with indegree 1 and outdegree 1 in $D$ ). Clearly, $\left(A_{2}, A^{-}, A^{+}\right)$is a partition of $V(D) \backslash\left\{v_{1}, v_{n}\right\}$. Observe that $\left|A_{2}\right|=n-n_{3}$. Since $\sum_{v \in V(D)} d^{+}(v)=\sum_{v \in V(D)} d^{-}(v)$, we have $\left|A^{+}\right|=\left|A^{-}\right|$, and so $\left|A^{+}\right|=\left(n_{3}-2\right) / 2$.

Now for $i=1$ to $n-1$, we enumerate the $p_{i}$ partial 3-edge-colourings of the arcs whose tail is in $\left\{v_{1}, \ldots, v_{i}\right\}$. For $i=1$, there are 6 such colourings, since $d^{+}\left(v_{1}\right)=3$.

Now, for each $i$, when we want to extend the partial colourings, two cases may arise.

- If $d_{D}^{-}\left(v_{i}\right)=1$, then we need to colour one or two arcs, and one colour (the one of the arc entering $v_{i}$ ) is forbidden, so there are at most 2 possibilities. Hence $p_{i} \leq 2 p_{i-1}$.

- If $d_{D}^{-}\left(v_{i}\right)=2$, then we need to colour one arc, and at least two colours (the ones of the arcs entering $v_{i}$ ) are forbidden, so there is at most one possibility. Hence $p_{i} \leq p_{i-1}$. 
At the end, all the edges of $G$ are coloured, and a simple induction shows that $c_{3}(G)=p_{n-1} \leq$ $6 \cdot 2^{\left|A_{2}\right|+\left|A^{+}\right|}=3 \cdot 2^{n-\frac{n_{3}}{2}}$.

In particular, for a connected cubic graph $G$, we obtain $c_{3}(G) \leq 3 \cdot 2^{n / 2}$. We now extend this result to $k$-regular graphs.

First, remark that every $k$-edge-colouring of a $k$-regular graph $G$ is precisely the union of $k$ (pairwise disjoint) perfect matchings of $G$. So, a bound on the number of perfect matchings in a $k$-regular graph yields to a bound on the number of $k$-edge-colourings of this graph. Computing the maximal number of perfect matchings in a graph is a classical problem in Graph Theory [7]. The best bound on graphs with degree constraints is due to Alon and Friedland [1]. They proved that a $k$-regular graph admits at most $(k !)^{\frac{n}{2 k}}$ distinct perfect matchings. So, as a $k$-edge-colouring is made of $k$ perfect matchings, a $k$-regular graph admits at most $(k !)^{\frac{n}{2}} k$-edge-colourings. Under the assumption that the graph is connected, we improve this bound.

Theorem 5. Let $G$ be a connected $k$-regular graph, with $k \geq 3$. Then $c_{k}(G) \leq k \cdot((k-1) !)^{n / 2}$.

Proof. First, observe that if a connected $k$-regular graph $G$ admits a $k$-edge-colouring, then every colour induces a perfect matching of $G$, and then $n$ is even. Furthermore, observe that $G$ is 2connected. Indeed, assume that $G$ has a cutvertex $x$ and admits a $k$-edge-colouring $c$. As $G$ has an even number of vertices, one of the connected components, say $C$, of $G-x$ has odd cardinality. A colour appearing on an edge between $x$ and a connected component of $G-x$ different from $C$ must form a perfect matching on $C$ which is impossible. So, $G$ is 2-connected.

Hence we can use the method of the proof of Theorem 4 and consider an $(s, t)$-ordering $v_{1}, \ldots, v_{n}$ of $G$ and $D$ the orientation of $G$ obtained from this ordering (i.e. $v_{i} v_{j} \in A(D)$ if and only if $v_{i} v_{j} \in$ $E(G)$ and $i<j)$. The analysis made in the proof of Theorem 4 yields $c_{k}(G) \leq \prod_{x \in V(G)}\left(d^{+}(x) !\right)$. For $i=1, \ldots, k-1$, we define $A_{i}=\left\{x \in V(G) \backslash\left\{v_{1}, v_{n}\right\}: d^{+}(x)=i\right\}$. It is clear that $\left(A_{i}\right)_{1 \leq i \leq k-1}$ form a partition of $V(G) \backslash\left\{v_{1}, v_{n}\right\}$. If we denote $\left|A_{i}\right|$ by $a_{i}$, then $c_{k}(G) \leq P:=k ! \prod_{i=1}^{k-1}(i !)^{a_{i}}$. Moreover $S_{1}:=\sum_{i=1}^{k-1} a_{i}=n-2$ (by counting the number of vertices of $G$ ) and $S_{2}:=\sum_{i=1}^{k-1} i \cdot a_{i}=k(n-2) / 2$ (by counting the number of arcs of $D-v_{1}$ ).

Let us now find the maximum value of $P$ under the conditions $S_{1}=n-2$ and $S_{2}=k(n-2) / 2$. If we can find $1<p \leq q<k-1$ with $a_{p} \neq 0$ and $a_{q} \neq 0$ (or $a_{p} \geq 2$ if $p=q$ ), then we decrease $a_{p}$ and $a_{q}$ by one and increase $a_{p-1}$ and $a_{q+1}$ by one. Doing this, $S_{1}$ and $S_{2}$ are unchanged and $P$ is multiplied by $\frac{q+1}{p}>1$. We repeat this operation as many times as possible and stop when (a) for every $i=2, \ldots, k-2, a_{i}=0$ or $(b)$ there exists $j \in\{2, \ldots, k-2\}$ such that for every $i=2, \ldots, k-2$ and $i \neq j, a_{i}=0$ and $a_{j}=1$. In case $(b), S_{1}$ gives $a_{1}+1+a_{k-1}=n-2$ and $S_{2}$ is $a_{1}+j+(k-1) a_{k-1}=k(n-2) / 2$. Combining $S_{1}$ and $S_{2}$, we obtain $2(k-2) a_{k-1}+2(j-1)=$ $(k-2)(n-2)$ and we conclude that $k-2$ divides $2(j-1)$ and so that $j=k / 2$. Solving $S_{1}$ and $S_{2}$ we have in particular that $a_{1}=a_{k-1}$ and $2 a_{1}=n-3$ which is impossible, as $n$ is even. Hence, we are in case $(a)$, and solving $S_{1}$ and $S_{2}$ yields to $a_{1}=a_{k}=(n-2) / 2$. We conclude that $P \leq k !((k-1) !)^{n / 2-1}$.

We turn now the proof of Theorem 5 into an algorithm to enumerate all the $k$-edge-colourings of a connected $k$-regular graph.

Corollary 6. There is an algorithm to enumerate all the $k$-edge-colourings of a connected $k$-regular graph on $n$ vertices in time $O^{*}\left(((k-1) !)^{n / 2}\right)$ and polynomial space. 
Proof. Let $G$ be a connected $k$-regular graph. We first check the 2-connectivity of $G$. If it is not 2 -connected, then we return 'The graph is not $k$-edge-colourable'.

If it is 2-connected, then we proceed as follows. We compute an $(s, t)$-ordering $v_{1}, \ldots, v_{n}$ of $G$, which can be done in polynomial time (see [8] and [9] for instance), and orient $G$ accordingly to this ordering. Now, it is classical to enumerate all the permutations of a set of size $p$ in time $O(p !)$ and linear space, in such way that, being given a permutation we compute in average constant time the next permutation in the enumeration (with the Steinhaus-Johnson-Trotter algorithm for instance, see $[15])$.

Using this and the odometer principle, it is now easy to enumerate all the edge colourings we want. In the enumeration of all the permutations of $\{1, \ldots, k\}$, we take the first one and assign the corresponding colours to the arc with tail $x_{1}$. For any index $i$ with $2 \leq i \leq k$, we assign to the arcs with tail $x_{i}$ the first permutation in the enumeration of the permutations of the possible colours for these arcs (i.e. all the colours of $\{1, \ldots, k\}$ minus the one of the arcs entering in $x_{i}$ ). Then, we have the first colouring, and we check if it is a proper edge colouring of $G$ (in polynomial time). To obtain the next colouring, we take the next permutation on the colours possible on the arcs with tail $x_{n-1}$, and so on. Once all the possible permutations have been enumerated for these arcs, we take the next permutation on the colours possible on the arcs with tail $x_{n-2}$ and re-enumerate the permutation of possible colours for the arcs with tail $x_{n-1}$, and so on, following the odometer principle.

The bound given by Theorem 5 is optimal on the class of connected $k$-regular graphs. For all $k \geq 3$, and $n \geq 2, n=2 p$ even, the $k$-noodle necklace $N_{n}^{k}$ is the $k$-regular graph obtained from a cycle on $2 p$ vertices $\left(v_{1}, v_{2}, \ldots, v_{2 p}, v_{1}\right)$ by replacing all the edges $v_{2 i-1} v_{2 i}, 1 \leq i \leq p$ by $k-1$ parallel edges.

Proposition 7. Let $k \geq 3$ and $n \geq 2$,

$$
c_{k}\left(N_{n}^{k}\right)=k \cdot((k-1) !)^{n / 2} .
$$

Proof. Observe that in every $k$-edge-colouring of $N_{n}^{k}$ the edges which are not multiplied (i.e. $\left.v_{2 i} v_{2 i+1}\right)$ are coloured the same. There are $k$ choices for such a colour. Once this colour is fixed, there are $(k-1)$ ! choices for each set of $k-1$ parallel edges. Hence $c_{k}\left(N_{n}^{k}\right)=k \cdot((k-1) !)^{n / 2}$.

\subsection{A more precise bound for cubic graphs}

For simple cubic graphs, we lower the bound on the number of 3 -edge-colourings from $3 \cdot 2^{n / 2}$ to $\frac{9}{4} \cdot 2^{n / 2}$.

Lemma 8. If $G$ is a connected cubic simple graph, then $c_{3}(G) \leq \frac{9}{4} \cdot 2^{n / 2}$.

Proof. As in Theorem 4, let us consider an $(s, t)$-ordering $v_{1}, v_{2}, \ldots, v_{n}$ of the vertices and the acyclic digraph $D$ obtained by orienting all the edges of $G$ according to this ordering.

Let $i$ be the smallest integer such that $d^{-}\left(v_{i}\right)=2$. Since every vertex (except $v_{1}$ ) has an inneighbour, there exists $j$ such that there are two internally-disjoint directed paths from $v_{j}$ to $v_{i}$ in $D$. In $G$, the union of these two paths forms a cycle $C$. By definition of $i$, all vertices of $C$ but $v_{i}$ have outdegree 2. So, if there is $k$ such that $j<k<i$ and $v_{k} \notin V(C)$, then $v_{k}$ has no outneighbour in $C$ and the ordering $v_{1}, \ldots, v_{k-1}, v_{k+1}, \ldots v_{i}, v_{k}, v_{i+1}, \ldots, v_{n}$ is also an $(s, t)$-odering. Repeating 
this operation as many times as necessary, we may obtain that all the vertices of $C$ are consecutive in the ordering, that is $C=\left(v_{j}, v_{j+1}, \ldots, v_{i}, v_{j}\right)$.

We enumerate the 3-edge-colourings of $G$ in a similar way to the proof of Theorem 4, except that instead of examining the colour of arcs with tail in $\left\{v_{j}, \ldots, v_{i}\right\}$ one after another, we look at $C$ globally. If $j=1$, then there are exactly $c_{3}(C)$ 3-edge-colourings of $C$, because no $\operatorname{arcs}$ has head in $C$. If $j>1$, then there are $c_{3}(C) / 33$-edge-colourings of $C$, because one arc has head $v_{j}$ and we need the colours of the two arcs with tail $v_{j}$ to have a colour distinct from it.

Recall that in $D, v_{1}$ has indegree $0, v_{p}$ has indegree $3, \frac{n-2}{2}$ vertices have indegree 2 and $\frac{n-2}{2}$ vertices have indegree 1 . If $j>1$ (resp. $j=1$ ), then there are $i-j$ (resp. $i-2$ ) vertices of indegree 1 in $C$, so there are $\frac{n-2 i+2 j-2}{2}$ (resp. $\frac{n-2 i+2}{2}$ ) vertices of indegree 1 in $V(G) \backslash V(C)$.

If $j=1$, then we start by colouring $C$ and then extend the colouring to $G$. Once $C$ is coloured, there is at most one possibility to colour each arc with tail in $C$, so $c_{3}(G) \leq c_{3}(C) \cdot 2^{\frac{n-2 i+2}{2}}=$ $\left(c_{3}(C) / 2^{i-1}\right) \cdot 2^{n / 2}$. If $j>1$, we colour the arcs with tail in $\left\{v_{1}, \ldots, v_{j-1}\right\}$ as usual. Remark that, by the choice of $C$, there is exactly one of these arcs, denoted by $e$, which has head in $C$ and more precisely $e$ has tail $v_{j}$. Then, we consider all the 3-edge-colourings of $C$ that agree with the colour of $e$ (i.e. $c\left(v_{j} v_{j+1}\right) \neq c(e)$ and $\left.c\left(v_{j} v_{i}\right) \neq c(e)\right)$. There are exactly $c_{3}(C) / 3$ such colourings. Finally, we extend the edge colourings in all possible ways to $G$ using the usual method. So, in this case, we obtain $c_{3}(G) \leq 6 \cdot c_{3}(C) / 3 \cdot 2^{\frac{n-2 i+2 j-2}{2}}=\left(c_{3}(C) / 2^{i-j}\right) \cdot 2^{n / 2}$.

In all cases, we have to bound the value $\frac{c_{3}(C)}{2^{i-j}}$ for $1 \leq i<j$. Since $G$ has no 2-cycles, $C$ has length at least 3 , and so $i-j \geq 2$. By Proposition $1, c_{3}(G)=2^{i-j+1}+2$ if $i-j$ is odd, and $c_{3}(G)=2^{i-j+1}-2$ if $i-j$ is even. Easily one sees that the value $\frac{c_{3}(C)}{2^{i-j}}$ is maximized when $i-j=3$ (i.e. $C$ has length four), and so $\frac{c_{3}(C)}{2^{i-j}} \leq \frac{18}{8}=\frac{9}{4}$. Thus $c_{3}(G) \leq \frac{9}{4} \cdot 2^{n / 2}$.

Theorem 5 for $k=3$ states that a connected cubic graph $G$ has at most $3 \cdot 2^{n / 2} 3$-edge-colourings. We shall now describe all connected cubic graphs attaining this bound.

Let $G$ be a cubic graph and $C=u v u$ be a 2 -cycle in $G$. Then $G / C$ is the graph obtained from $G-\{u, v\}$ by adding an edge between the neighbour of $u$ distinct from $v$ and the neighbour of $v$ distinct from $u$.

Let $\Theta$ be the graph with two vertices joined by three edges. And let $\mathcal{L}$ be the family of graphs defined recursively as follows:

- $\Theta \in \mathcal{L}$.

- if $G$ has a 2-cycle $C$ such that $G / C$ is in $\mathcal{L}$, then $G$ is in $\mathcal{L}$.

Remark that the 3-noodle necklaces $N_{n}^{3}$ (with $n$ even) belongs to the family $\mathcal{L}$.

Theorem 9. Let $G$ be a connected cubic graph. If $G \in \mathcal{L}$, then $c_{3}(G)=3 \cdot 2^{n / 2}$. Otherwise $c_{3}(G) \leq \frac{9}{4} \cdot 2^{n / 2}$.

Proof. By induction on $n$, the result holding for simple graphs by Lemma 8 and for $\Theta$ because $c_{3}(\Theta)=6$.

Assume that $n \geq 4$ and that $G$ has a 2-cycle $C=u v u$. In any 3-edge-colouring of $G$, the edges not in $C$ incident to $u$ and $v$ are coloured the same. Hence to each 3-edge-colouring $c$ of $G / C$ corresponds the two 3-edge-colourings of $G$ that agrees with $c$ on $G-\{u, v\}$. Hence $c_{3}(G)=2 c_{3}(G / C)$. 
If $G / C$ is in $\mathcal{L}$, then $G$ is also in $\mathcal{L}$. Moreover, by the induction hypothesis, $c_{3}(G / C)=3 \cdot 2^{(n-2) / 2}$. So $c_{3}(G)=3 \cdot 2^{n / 2}$.

If $G / C$ is not in $\mathcal{L}$, then $G$ is not in $\mathcal{L}$. Moreover, by the induction hypothesis, $c_{3}(G / C) \leq$ $\frac{9}{4} \cdot 2^{(n-2) / 2}$. So $c_{3}(G) \leq \frac{9}{4} \cdot 2^{n / 2}$.

We have no example of cubic simple graphs admitting exactly $\frac{9}{4} \cdot 2^{n / 2} 3$-edge-colourings, and we believe that $\frac{9}{4}$ could be replaced by a lower constant in the statement of Theorem 9 . In fact, we conjecture that the maximum number of 3 -edge-colourings of cubic simple graphs of order $n$ is attained by some special graphs that we now describe.

For all $n \geq 2, n=2 p$ even, the hamster wheel $H_{n}$ is the cubic graph obtained from two cycles on $p$ vertices $C_{v}=\left(v_{1}, v_{2}, \ldots, v_{p}, v_{1}\right)$ and $C_{w}=\left(w_{1}, w_{2}, \ldots, w_{p}, w_{1}\right)$ by adding the matching $M=\left\{v_{i} w_{i}: 1 \leq i \leq p\right\}$. This construction for a lower bound was proposed by Pyatkin as it is mentioned in [12].

Proposition 10.

$$
c_{3}\left(H_{n}\right)= \begin{cases}2^{n / 2}+8, & \text { if } n / 2 \text { is even, } \\ 2^{n / 2}-2, & \text { if } n / 2 \text { is odd }\end{cases}
$$

Proof. Let $\phi$ is a 3-edge-colouring of $C_{v}$.

If the three colours appear on $C_{v}$, then there is a unique 3-edge-colouring of $H_{n}$ extending $\phi$. Indeed, to extend $\phi$, the colours of the edges of $M$ are forced. Since the three colours appear on $C_{v}$, there are two edges of $M$ which are coloured differently. Without loss of generality, we may assume that these two edges are consecutive, that is there exists $i$ such that they are $v_{j} w_{j}$ and $v_{j+1} w_{j+1}$. But then the colour of $w_{j} w_{j+1}$ must be equal to the one of $v_{j} v_{j+1}$. Then, from edge to edge along the cycle, one shows that for all $i$, the colour of $w_{i} w_{i+1}$ is the one of $v_{i} v_{i+1}$.

If only two colours appear on $C_{v}$, then there are two 3 -edge-colourings of $H_{n}$ extending $\phi$. Indeed in this case, $n$ is even and all the edges of $M$ must be coloured by the colour not appearing on $C_{v}$. So, there are two possible 3 -edge-colourings of $C_{w}$ with the colours appearing on $C_{v}$.

Hence the number of 3-edge-colourings of $G$ is equal to the number of 3-edge-colourings of $C_{v}$ plus the number of 3-edge-colourings of $C_{v}$ in which two colours appear. If $n / 2$ is odd, this last number is 0 , and if $n / 2$ is even, this number is 6 . So, by Proposition $1, c_{3}\left(H_{n}\right)=2^{n / 2}-2$ if $n / 2$ is odd and $c_{3}\left(H_{n}\right)=2^{n / 2}+8$ if $n / 2$ is even.

For all $n \geq 2, n=2 p$ even, the Mobius ladder $M_{n}$ is the cubic graph obtained from a cycle on $n$ vertices $C=\left(v_{1}, v_{2}, \ldots v_{n}, v_{1}\right)$ by adding the matching $M=\left\{v_{i} v_{i+p}: 1 \leq i \leq p\right\}$ (indices are modulo $n$ ).

Two edges $e$ and $f$ of the cycle $C$ are said to be antipodal, if there exists $1 \leq i \leq p$ such that $\{e, f\}=\left\{v_{i} v_{i+1}, v_{i+p} v_{i+p+1}\right\}$. A 3-edge-colouring $c$ of $M_{n}$ is said to be antipodal if $c(e)=c(f)$ for every pair $(e, f)$ of antipodal edges.

Proposition 11. Let $c$ be a 3-edge-colouring of $M_{n}$. If $c$ is not antipodal, then $n / 2$ is odd and all the arcs $v_{i} v_{i+n / 2+1}$ are coloured the same.

Proof. Suppose that two antipodal edges are not coloured the same. Without loss of generality, $c\left(v_{n} v_{1}\right)=2$ and $c\left(v_{p} v_{p+1}\right)=3$, where $n=2 p$. Hence, we have $c\left(v_{1} v_{p+1}\right)=1, c\left(v_{1} v_{2}\right)=3$ and $c\left(v_{p+1} v_{p+2}\right)=2$. And so on by induction, for all $1 \leq i \leq p, c\left(v_{i} v_{i+p}\right)=1$ and $\left\{c\left(v_{i} v_{i+1}\right), c\left(v_{i+p} v_{i+p+1}\right)\right\}=$ $\{2,3\}$. Hence, the edges of $C$ are coloured alternately with 2 and 3 , Since $c\left(v_{2 p} v_{1}\right)=2$ and $c\left(v_{p} v_{p+1}\right)=3, p$ must be odd. 


\section{Proposition 12.}

$$
c_{3}\left(M_{n}\right)= \begin{cases}2^{n / 2}+2, & \text { if } n / 2 \text { is even }, \\ 2^{n / 2}+4, & \text { if } n / 2 \text { is odd. }\end{cases}
$$

Proof. Clearly, there is a one-to-one mapping between the antipodal 3-edge-colourings of $M_{n}$ and the 3-edge-colourings of $C_{n / 2}$. Hence, by Proposition 11, if $n / 2$ is even, then $c_{3}\left(M_{n}\right)=c_{3}\left(C_{n / 2}\right)=$ $2^{n / 2}+2$ by Proposition 1.

If $n / 2$ is odd, non-antipodal 3-edge-colourings are those such that all $\operatorname{arcs} v_{i} v_{i+n / 2+1}$ are coloured the same, by Proposition 11. There are 6 such edge colourings (three choices for the colour of the edges $v_{i} v_{i+n / 2+1}$ and for each of these choices, two possible edge colourings of $C$ ). Hence $c_{3}\left(M_{n}\right)=c_{3}\left(C_{n} / 2\right)+6=2^{n / 2}+4$ by Proposition 1 .

We think that $H_{n}$ and $M_{n}$ are the connected cubic graphs which admit the maximum number of 3-edge-colourings. Precisely, we raise the following conjecture.

Conjecture 13. Let $G$ be a connected cubic simple graph on $n$ vertices. If $n / 2$ is even, then $c_{3}(G) \leq c_{3}\left(H_{n}\right)$ and if $n / 2$ is odd, then $c_{3}(G) \leq c_{3}\left(M_{n}\right)$.

\section{Total colouring}

A total colouring of a graph $G$ into $k$ colours is a colouring of its vertices and edges such that two adjacent vertices receive different colours, two adjacent edges receive different colours and a vertex and an edge incident to it receive different colours. A total colouring with $k$ colours is a $k$-total-colouring. For every graph $G$, let $c_{k}^{T}(G)$ be the number of $k$-total-colourings of $G$.

For each 4-edge-colouring $c$ of a cubic graph $G$, there is at most one 4-total-colouring of $G$ whose restriction to $E(G)$ equals $c$. Indeed, the colours of the three edges incident to a vertex force the colour of this vertex. Hence if $G$ is cubic, we have that $c_{4}^{T}(G) \leq c_{4}(G)$.

By the method described in the previous section, one can show that if $G$ is 2-connected, then $c_{4}(G)=O\left(2^{n / 2} \cdot 6^{n / 2}\right)$, and so $c_{4}^{T}(G)=O\left(2^{n / 2} \cdot 6^{n / 2}\right)$. We now obtain better upper bounds for $c_{4}^{T}$.

Theorem 14. Let $G$ be a 2-connected subcubic graph. Then $c_{4}^{T}(G) \leq 3 \cdot 2^{2 n-n_{3} / 2}$.

Proof. Assume first that $G$ is a cycle $\left(v_{1}, \ldots, v_{n}, v_{1}\right)$. Let us totally colour it greedily starting from $v_{1}$. There are 4 possible colours for $v_{1}$, and then 3 possible colours for $v_{1} v_{2}$. Afterwards for every $i \geq 2$, there at most two possible colours for $v_{i}$ (the ones distinct from the colours of $v_{i-1}$ and $v_{i-1} v_{i}$ ) and then at most two possible colours for $v_{i} v_{i+1}$ (the ones distinct from the colours of $v_{i-1} v_{i}$ and $\left.v_{i}\right)$. Hence $c_{4}^{T}(G) \leq 4 \cdot 3 \cdot 2^{2 n-2}=3 \cdot 2^{2 n}$.

Assume now that $G$ is not a cycle. Let $s$ and $t$ be two distinct vertices of degree 3. Consider an $(s, t)$-ordering $v_{1}, v_{2}, \ldots, v_{n}$ of $V(G)$, which exists by Lemma 3 , and the orientation $D$ of $G$ according to this ordering. Then $d^{+}\left(v_{1}\right)=3=d^{-}\left(v_{n}\right)$ and $d^{-}\left(v_{1}\right)=0=d^{+}\left(v_{n}\right)$. Let $A^{+}$(resp. $A^{-}$) be the set of vertices of outdegree 2 (resp. indegree 2) in $D$ and $A_{2}$ be the set of vertices of degree 2 in $G$. As in the proof of Theorem 4, we have $\left|A_{2}\right|=n-n_{3}$, and $\left|A^{+}\right|=\left|A^{-}\right|=\left(n_{3}-2\right) / 2$.

Now for $i=1$ to $n-1$, we enumerate the $p_{i}$ partial 4 -total-colourings of vertices in $\left\{v_{1}, \ldots, v_{i}\right\}$ and arcs with tail in $\left\{v_{1}, \ldots, v_{i}\right\}$. For $i=1$, there are $4 !=24$ such colourings, since $v_{1}$ and its three incident arcs must receive different colours.

For each $1<i<n$, when we extend the partial total colourings. Two cases may arise. 
- If $d_{D}^{-}\left(v_{i}\right)=1$, then there are two choices to colour $v_{i}$ and then two other choices to colour the (at most two) arcs leaving $v_{i}$. Hence $p_{i} \leq 4 p_{i-1}$.

- If $d_{D}^{-}\left(v_{i}\right)=2$, then there are at most two choices to colour $v_{i}$ and then the colour of the arc leaving $v_{i}$ is forced since three colours are forbidden by $v_{i}$ and its two entering arcs. Hence $p_{i} \leq 2 p_{i-1}$.

Finally, we need to colour $v_{n}$. Since its three entering arcs are coloured its colour is forced (or it is impossible to extend the colouring).

Hence an easy induction shows that $c_{4}^{T}(G)=p_{n-1} \leq 24 \cdot 4^{\left|A_{2}\right|+\left|A^{+}\right|} \cdot 2^{\left|A^{-}\right|}=3 \cdot 2^{2 n-n_{3} / 2}$.

A leaf of a tree is a degree one vertex. A vertex of a tree which is not a leaf is called a node. A tree is binary if all its nodes have degree 3 .

Proposition 15. If $T$ is a binary tree of order $n$, then $c_{4}^{T}(T)=3 \cdot 2^{3 n / 2}$.

Proof. By induction on $n$, the results holding easily when $n=2$, that is when $T=K_{2}$.

Suppose now that $T$ has more than two vertices. There is a node $x$ which is adjacent to two leaves $y_{1}$ and $y_{2}$. Consider the tree $T^{\prime}=T-\left\{y_{1}, y_{2}\right\}$. By the induction hypothesis, $c_{4}^{T}\left(T^{\prime}\right)=3 \cdot 2^{3(n-2) / 2}$. Now each 4-total-colouring of $T^{\prime}$ may be extended into exactly eight 4-total-colourings of $T^{\prime}$. Indeed the two colours of $x$ and its incident edge in $T^{\prime}$ are forbidden for $x y_{1}$ and $x y_{2}$, so there are two possibilities to extend the colouring to these edges, and then for each $y_{i}$, there are two possible colours available. Hence $c_{4}^{T}(T)=8 \cdot c_{4}^{T}\left(T^{\prime}\right)=3 \cdot 2^{3 n / 2}$.

Theorem 16. Let $G$ be a connected cubic graph. Then $c_{4}^{T}(G) \leq 3 \cdot 2^{3 n / 2}$.

Proof. Let $F$ be the subgraph induced by the cutedges of $G$. Then $F$ is a forest. Consider a tree of $F$. It is binary, its leaves are in different non-trivial 2-connected components of $G$, and every node is a trivial 2-connected component of $G$.

A subgraph $H$ of $G$ is full if it is induced on $G$, connected and such that for every non-trivial 2-connected component $C, H \cap C$ is empty or is $C$ itself and for every tree $T$ of $F, H \cap T$ is empty, or is just one leaf of $T$ or is $T$ itself. Observe that a full subgraph has minimum degree at least 2.

We shall prove that for every full subgraph $H, c_{4}^{T}(H) \leq 3 \cdot 2^{2 n(H)-n_{3}(H) / 2}$. We proceed by induction on the number of 2-connected components of $H$. If $H$ is 2-connected, then the result holds by Theorem 14 .

Suppose now that $H$ is not 2-connected. Then $H$ contains a tree $T$ of $F$. Let $v_{1}, \ldots, v_{p}$ be the leaves of $T, e_{i}, 1 \leq i \leq p$ the edge incident to $v_{i}$ in $T$ and $N$ the set of nodes of $T$. Then $H-N$ has $p$ connected components $H_{1}, \ldots, H_{p}$ such that $v_{i} \in H_{i}$ for all $1 \leq i \leq p$. Furthermore, each $H_{i}$ is a full subgraph of $G$.

Let $c$ be a 4-total-colouring of $T$. It can be extended to $H_{i}$ by any 4-total-colouring of $H_{i}$ such that $v_{i}$ is coloured $c\left(v_{i}\right)$ and the two edges incident to $v_{i}$ in $H_{i}$ are coloured in $\{1,2,3,4\} \backslash$ $\left\{c\left(v_{i}\right), c\left(e_{i}\right)\right\}$. There are $\frac{1}{12} c_{4}^{T}\left(H_{i}\right)$ such colourings because each of them correspond to exactly twelve 4 -total colourings of $H_{i}$ obtained by permuting the colour of $v_{i}$ (there are 4 possibilities) and then the colour of $e_{i}$ (there are 3 possibilities). Hence each 4-total-colouring of $T$ can be extended into $\prod_{i=1}^{p} \frac{1}{12} c_{4}^{T}\left(H_{i}\right)$ 4-total-colourings of $H$ and so

$$
c_{4}^{T}(H)=c_{4}^{T}(T) \cdot \prod_{i=1}^{p} \frac{1}{12} c_{4}^{T}\left(H_{i}\right) .
$$


Now by Proposition 15, $T$ has $3 \cdot 2^{3 n(T) / 2}$ 4-total-colourings, and since $H_{i}$ is full $c_{4}^{T}\left(H_{i}\right) \leq 3$. $2^{2 n\left(H_{i}\right)-n_{3}\left(H_{i}\right) / 2}$ by the induction hypothesis. Moreover, $n_{3}(H)=n(T)+\sum_{i=1}^{p} n_{3}\left(H_{i}\right)$ and $n(H)=$ $n(T)+\sum_{i=1}^{p} n\left(H_{i}\right)-p$. Hence $c_{4}^{T}(H) \leq 3 \cdot 2^{2 n(H)-n_{3}(H) / 2}$.

As previously for the edge colourings of graphs, we derive from Theorem 16 an algorithm to enumerate all the 4-total-colourings of a cubic graph. The proof is similar to the one of Corollary 6 .

Corollary 17. There is an algorithm to enumerate all the 4-total-colourings of a connected cubic graph on $n$ vertices in time $O^{*}\left(2^{3 n / 2}\right)$ and polynomial space.

The bound of Theorem 16 is seemingly not tight. Indeed, in Theorem 14, the equation $p_{i} \leq 2 p_{i-1}$ when $d_{D}^{-}\left(v_{i}\right)$ often overestimates $p_{i}$, because there are two choices to colour $v_{i}$ only if the two colours appearing on its two entering arcs are the same two as the ones assigned to the tails of these arcs. If not the colour of $v_{i}$ is forced or $v_{i}$ cannot be coloured.

Problem 18. What is $c_{4}^{T}(n)$, the maximum of $c_{4}^{T}(G)$ over all connected graphs of order $n$ ?

We shall now give a lower bound on $c_{4}^{T}(n)$. A binary tree is nice if its set of leaves may be partitionned into pairs of twins, i.e. leaves at distance 2. Clearly, every nice binary tree $T$ has an even number of leaves and thus $n(T) \equiv 2 \bmod 4$. Moreover if $n(T)=4 p+2$, then $T$ has $2 p$ nodes and $p+1$ pairs of twins. A noodle tree is a cubic graph obtained from a nice binary tree by adding two parallel edges between each pair of twins.

Proposition 19. Let $p$ be a positive integer and $n=4 p+2$. If $G$ is a noodle tree $G$ of order $n$, then $c_{4}^{T}(G)=\frac{3}{\sqrt{2}} \cdot 2^{5 n / 4}$.

Proof. Let $X_{1}, \ldots, X_{p+1}$ be the pairs of twins of $G$, and let $T$ be the binary tree $G-\bigcup_{i=1}^{p+1} X_{i}$. Let us label the leaves of $T, y_{1}, \ldots, y_{p+1}$ such that for all $1 \leq i \leq p+1, y_{i}$ is adjacent to the two vertices of $X_{i}$ in $G$.

Every 4 -total-colouring of $T$, may extended in exactly 4 ways to each pair of twins $X_{i}=\left\{x_{i}, x_{i}^{\prime}\right\}$ and their incident edges. Indeed, without loss of generality we may assume that $y_{i}$ is coloured 1 and its incident edge in $T$ is coloured 2. Then the edges $y_{i} x_{i}$ and $y_{i} x_{i}^{\prime}$ must be coloured in $\{3,4\}$, which can be done in two possible ways. For each of these possibilities, the parallel edges between $x_{i}$ and $x_{i}^{\prime}$ must be coloured in $\{1,2\}$, which again can be done in two possible ways. Finally, we must colour $x_{i}$ (resp. $x_{i}^{\prime}$ ) with the colour of $y_{i} x_{i}^{\prime}$ (resp. $\left.y_{i} x_{i}\right)$.

Hence $c_{4}(G)=4^{p+1} \cdot c_{4}(T)$, and so by Proposition $15, c_{4}(G)=3 \cdot 2^{5 p+2}$.

\section{Acknowledgement}

The authors would like to thank their children for suggesting them some graph names.

\section{References}

[1] N. Alon and S. Friedland. The maximum number of perfect matchings in graphs with a given degree sequence. Electr. J. Comb. 15, N13, 2008.

[2] R. Beigel and D. Eppstein. 3-coloring in time $O\left(1.3289^{n}\right)$. Journal of Algorithms 54:168-204, 2005. 
[3] A. Björklund and T. Husfeldt. Inclusion-exclusion algorithms for counting set partitions. In Proceedings of the 47th Annual IEEE Symposium on Foundations of Computer Science (FOCS 2006), pp. 575-582. IEEE, Los Alamitos, 2006.

[4] A. Björklund, T. Husfeldt, P. Kaski, and M. Koivisto. Narrow sieves for parameterized paths and packings. arXiv:1007.1161v1, 2010.

[5] A. Björklund, T. Husfeldt, and M. Koivisto. Set partitioning via Inclusion-Exclusion. SIAM J. Comput., 39(2):546-563, 2009.

[6] J. A. Bondy and U. S. R. Murty. Graph theory. Graduate Texts in Mathematics 244, Springer, 2008 (2nd ed.).

[7] L.M. Bregman. Some properties of nonnegative matrices and their permanents. Soviet Math. Dokl., 14:945-949, 1973.

[8] S. Even and R. E. Tarjan. Computing an st-numbering. Theoretical Computer Science, $2(3): 339-344,1976$.

[9] S. Even and R. E. Tarjan. Corrigendum: Computing an st-numbering. Theoretical Computer Science, 4(1):123, 1977.

[10] F. V. Fomin, S. Gaspers, and S. Saurabh. Improved exact algorithms for counting 3- and 4-colorings. In Lin, G. (ed.) COCOON 200\%, Lecture Notes in Computer Science 4598:65-74, 2007.

[11] M. R. Garey and D. S. Johnson, Computers and intractability. A guide to the theory of NPcompleteness. A Series of Books in the Mathematical Sciences. W. H. Freeman and Co., San Francisco, Calif., 1979.

[12] P. A. Golovach, D. Kratsch, and J. F. Couturier. Colorings with few colors: counting, enumeration and combinatorial bounds. In Proceedings of WG 2010, Lecture Notes in Computer Science 6410:39-50, 2010.

[13] I. Holyer. The NP-completeness of edge-colouring. SIAM J. Computing 2:225-231, 1981.

[14] T. R. Jensen and B. Toft. Graph coloring problems. Wiley-Interscience Series in Discrete Mathematics and Optimization. John Wiley \& Sons, Inc., New-York, 1995.

[15] D. E. Knuth. The art of Computer Programming, volume 4A: Combinatorial Algorithms, Part 1. Addison-Wesley Professional, 2011.

[16] M. Koivisto. An $O\left(2^{n}\right)$ algorithm for graph coloring and other partitioning problems via inclusion-exclusion. In Proceedings of the 47th Annual IEEE Symposium on Foundations of Computer Science (FOCS 2006), pp. 583-590. IEEE, Los Alamitos, 2006.

[17] L. Kowalik. Improved edge-coloring with three colors. Theoret. Comp. Sci. 410:3733-3742, 2009.

[18] A. Lempel, S. Even, and I. Cederbaum. An algorithm for planarity testing of graphs. In P. Rosenstiehl, editor, Proceedings of the International Symposium on the Theory of Graphs (Rome, July 1966): 215-232. Gordon and Breach, 1967. 
[19] A. Sánchez-Arroyo. Determining the total colouring number is NP-hard. Discrete Math. 78(3):315-319, 1989. 